\section{Bei Vitiligo-Kindern an eine mögliche Thyreoiditis denken?}

\author{
Für die Vitiligo nimmt man eine autoimmune Genese an. Eine Assoziation mit \\ diversen Autoimmunerkrankungen wie Morbus Addison, Lupus erythematodes, \\ perniziöser Anämie sowie insbesondere mit Hashimoto-Thyreoiditis ist bekannt. \\ Nicht so klar ist die Sachlage bezüglich einer solchen Korrelation bei Vitiligo im \\ Kindesalter - in der Literatur finden sich hierzu widersprüchliche Daten.
}

munkrankheit oder beidem. Eine offenkundige Hypo- oder Hyperthyreose wurde bei keinem der 100 Kinder festgestellt. In der Vitiligo-Gruppe wiesen fünf, bei den Kontrollen sechs Kinder eine subklinische Unterfunktion der Schilddrüse auf.

Bei vier Mädchen (8\%) mit der Pigmentstörung - alle mit klinischem Vulgaris-Typ - wies man erhöhte Anti-TPOLevel nach, dagegen bei keiner der Kontrollpersonen. Damit ergab sich hierfür ein Verhältnis von 8 versus $0 \%(p=0,041)$. In allen vier Fällen wurde die Diagnose Hashimoto-Thyreoiditis sonografisch bestätigt. Eines der betroffenen Kinder erhielt bereits L-Thyroxin substituiert, ein anderes hatte eine subklinische Schilddrüsen-Unterfunktion. Die zwei anderen waren euthyreot.

Die Assoziation mit der AutoimmunThyreoiditis war signifikant mit dem weiblichen Geschlecht assoziiert; alle vier Mädchen mit der Thyreoiditis litten bereits deutlich länger als die anderen unter der Vitiligo.

Fazit: Wegen der erhöhten Inzidenz von Hashimoto-Thyreoiditiden in ihrer Kohorte empfehlen die Autoren, Kinder und Jugendliche mit Vitiligo, insbesondere Mädchen und speziell bei generalisiertem oder Vulgaris-Typ, jährlich einem Schilddrüsen-Screening zu unterziehen sowie Schilddrüsenantikörper bestimmen zu lassen, damit gegebenenfalls frühzeitig behandelt werden kann.

Waltraud Paukstadt

Uncu S et al. Relevance of autoimmune thyroiditis in children and adolescents with vitiligo. Int J Dermatol 2011; 50: 175-9

\section{Orale Hyposensibilisierung bei Erdnussallergie?}

Über erste Erfahrungen mit einer oralen Immuntherapie bei Erdnussallergie berichtete jetzt eine Arbeitsgruppe um Katharina Blümchen aus der Berliner Charité.

K inderärzte aus Berlin und New York untersuchten 23 Kinder zwischen drei und 14 Jahren, die eine Erdnussallergie im doppelblinden und placebokontrollierten Provokationstest (DBPCFC) und im IgE-RAST hatten. Die Hyposensibilisierung in Form von gerösteten Erdnüssen wurde mit einem siebentägigen Rush-Protokoll eingeleitet, bei Nichterreichen der protektiven Dosis von mindestens 0,5 g erhielten die Kinder eine Langzeit-Titration mit Dosissteigerungen alle zwei Wochen. Die Erhaltungsphase betrug acht Wochen und nach zweiwöchiger Karenz wurden die Patienten nochmals per DBPCFC provoziert.

Im Ergebnis tolerierten die Kinder nach der Rush-Aufdosierung über sieben Tage im Median lediglich eine Dosis von 0,15 g Erdnuss. 22 der 23 Patienten bekamen daher die Langzeitaufdosierung. Nach sieben Monaten erreichten 14 Patienten die anvisierte Schutzdosis von $0,5 \mathrm{~g}$ Erdnuss, im abschließenden DBPCFC wurde im Median $1 \mathrm{~g}(0,25-4 \mathrm{~g})$ vertragen, vor der oralen Immuntherapie waren es nur $0,19 \mathrm{~g}(0,02-1 \mathrm{~g})$.

Die Nebenwirkungen waren erträglich: bei $2,6 \%$ von insgesamt 6.137 verabreichten Einzeldosen fielen sie leicht bis mäßig aus. Nur bei vier der 22 Kinder musste die Behandlung abgebrochen werden. Im Labor zeigte sich ein Anstieg von erdnussspezifischem IgG4 und die Konzentration der Interleukine IL-5, IL-4 und IL-2 war danach vermindert.

Kommentar: Patienten mit schwerer Erdnussallergie müssen sich immer vor anaphylaktischen Reaktionen fürchten. Ein möglicher Schutz könnte eine orale Immunisierung bieten. Eine - zugegeben aufwendige - Hyposensibilisierung führt offensichtlich bei über der Hälfte von ih-

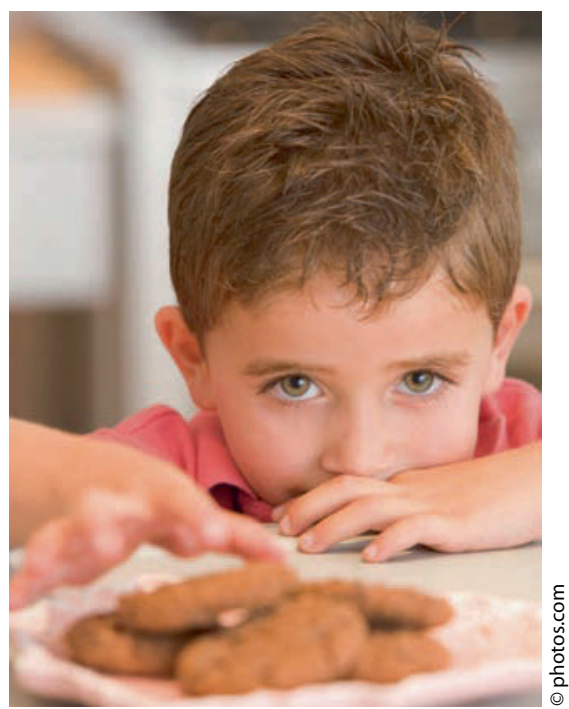

Nach einer Immuntherapie muss man keine Angst mehr vor Erdnussspuren in Lebensmitteln haben.

nen zur ausreichenden Toleranz gegen Erdnuss. Dr. Ulrich Mutschler

Blümchen $\mathrm{K}$ et al. Oral peanut immunotherapy in children with peanut anaphylaxis. J Allergy Clin Immunol 2010; 126: 83-91 